\title{
Cómo abordar la situación de marginación entre pares femeninos. Un estudio de caso en el tercer ciclo de Educación Primaria
}

\section{How to deal with the situation of isolation among female peers. A case study in the third cycle of Primary Education}

\author{
Raquel SÁNCHEZ RUIZ y Teresa ESCRIBANO LARA \\ Universidad de Castilla-La Mancha
}

Recibido: Febrero 2014

Aceptado: Abril 2014

\begin{abstract}
Resumen
Las burlas, el maltrato, el acoso y el bullying escolares se han convertido en un tema, por desgracia, habitual e incluso fomentado por las nuevas tecnologías. Y aunque su prevención y su tratamiento puedan ser dificultosos, es necesario que se aúnen los esfuerzos de padres, profesores, personal educativo y, en definitiva, toda la comunidad educativa para crear una atmósfera de cooperación y solventar cualquier problema de este tipo que pueda surgir.

Con estas premisas, los objetivos de este trabajo, que estudia un caso real de aislamiento sufrido por una alumna de tercer ciclo de Educación Primaria por parte de sus compañeras, son dos: por un lado, establecer un protocolo para prevenir casos similares; y por otro lado, engrosar la investigación al respecto y compendiar un protocolo de intervención que sirva como apoyo a la actuación institucional y establecer buenas prácticas para apoyar la praxis docente y la intervención de la comunidad educativa ante este tipo de situaciones.
\end{abstract}

Palabras clave: pares, alumnas, conducta antisocial, marginación.

\begin{abstract}
School teasing, abuse, violence, harassment and bullying unfortunately have become a current topic and have even been promoted by the use of new technologies. Although their prevention and processing may be difficult, it is necessary to join the efforts of parents, teachers, and education staff, in short, the whole educational community so as to create a cooperation atmosphere and solve any of these problems that might arise.

Under these premises, this paper, which studies a real isolation case suffered by a school girl in the third cycle of Primary Education and caused by a group of other school girls of the same class, has two main purposes: on the one hand, to establish a prevention protocol which might help avoid or tackle similar situations; on the other hand, to contribute to the investigation of school isolation and establish an action plan or intervention protocol that might support institutional action and disseminate the findings to support the teaching practice and the educational community intervention to face and tackle these situations.
\end{abstract}

Keywords: peers, girl students, antisocial behavior, marginalization. 
Tal y como afirma Freedman (2000, 1), las burlas pueden ocurrir en cualquier parte y su prevención es dificultosa a pesar de los esfuerzos de padres, profesores y personal educativo para crear una atmósfera de cooperación (Ross, 1996). Aunque existen muchos tipos de burlas y comportamientos de acoso y marginación, en este trabajo nos vamos a centrar en la marginación que se pueda producir entre compañeras dentro del aula del tercer ciclo de Primaria. Puesto que, si bien existen numerosos trabajos que analizan -especialmente en Educación Secundaria- el maltrato escolar (Díaz-Aguado Jalón, Martínez Arias y Martín Seoane, 2004; Haro-Solís, García-Cabrero y ReidlMartínez, 2013; Ramírez-López y Arcila-Rodríguez, 2013), la intimidación (Avilés Martínez y Monjas Casares, 2005), el bullying (Avilés Martínez, 2006; Brighi, Guarini, Melotti, Galli y Genta, 2012) y el ciberacoso (Garaigordobil y Aliri, 2013), el rol del propio maestro o profesor ante estas situaciones (Chagas Dorrey, 2005) o las conductas violentas en edades tempranas (Alsaker y Nagele, 2008; Alsaker y Vilén, 2010; Monks, Palermiti, Ortega y Costabile, 2011; Albaladejo-Blázquez, Ferrer-Cascales, Reig-Ferrer y Fernández-Pascual, 2013), ningún estudio ha abordado los procesos y las actuaciones en aquellas situaciones en las que un grupo de chicas marginan a otra u otras en Educación Primaria. Es más, como la propia Toldos $(2005,14)$ afirma, pese a las numerosas revisiones sobre las diferencias sexuales en cuanto a la agresión, poco se ha escrito sobre las diversas formas de comportamiento agresivo de las mujeres o adolescentes españolas; tan solo en los últimos años, algunas investigaciones abordan este tema en relación a la población española comparándola con otras culturas (Theron et al., 2001). Por el contrario, sí son frecuentes y recientes los estudios que investigan las diferencias de sexo en cuanto a la victimización femenina (Wade y Beran, 2011; Olenik-Shemesh, Heiman y Eden, 2012; Fenaughty y Harré, 2013).

Por tanto, el objetivo de este trabajo es identificar y definir un problema de aislamiento sufrido por una alumna del tercer ciclo de Educación Primaria por parte de sus compañeras dentro de un centro educativo real con el fin de difundir los resultados para, por un lado, prevenir casos similares y, por otro, engrosar la investigación al respecto y compendiar un protocolo de buenas prácticas y de actuación institucional para apoyar la praxis docente y la intervención de la comunidad educativa ante este tipo de situaciones.

\section{Marco teórico y metodología}

Entendemos por maltrato escolar cuando un alumno es agredido o es víctima de la exposición repetida y continuada a acciones negativas por parte de otro alumno o un conjunto de ellos (Olweus, 1998, 25), como Avilés Martínez (2006) afirma "con la intención de humillar y someter abusivamente a una víctima indefensa” (p. 82). No obstante, si bien nuestro estudio está vinculado de alguna manera con lo que se concibe como bullying o relaciones buscadas de dominio-sumisión que suelen implicar daño físico, humillación psicológica y/o aislamiento grupal (Avilés Martínez y Monjas Casares, 2005, 27), no lo abarca en su totalidad en tanto se centra únicamente en el aislamiento, y no en el resto de características, y en uno de los sexos -el femenino-, y no en pares escolares. En concreto, nuestro trabajo trata el tipo de maltrato entre iguales que Celaá $(2011,10)$ clasifica como “exclusión y marginación social” y sus 
subtipos: a) ignorar a alguien (pasiva); b) no dejarle participar (activa); y c) discriminar por razón de sexo, raza, discapacidad o cualquier otro motivo. Sin embargo, como decíamos, nos centraremos en el sexo femenino que, como Carretero Bermejo (2011, 38) afirma, suelen tener y abusar de un poder más social que físico.

Trautmann (2008, pp. 14-15) define los siguientes tres roles en las situaciones de acoso escolar, con los que coinciden otros autores aquí mencionados, como Carretero Bermejo (2011, 28) y Celaá $(2011,11)$, entre otros:

1) Agresor, que habitualmente proviene de entornos familiares con dificultades, con escasas normas de disciplina y/o límites. A veces, tales familias mezclan la permisividad excesiva ante conductas inadecuadas o antisociales con métodos muy autoritarios y coercitivos, donde se incluye frecuentemente el castigo físico (Díaz-Aguado Jalón et al., 2004). Igualmente, son físicamente más fuertes que sus pares, dominantes, impulsivos, con baja tolerancia a la frustración, desafiantes a la autoridad y con actitud positiva hacia la violencia. Salmivalli (1999, 454) divide este rol en los siguientes subtipos: a) agresor líder (bully), es decir, aquellos que inician la agresión en las situaciones de acoso escolar entre iguales; b) agresor seguidor (assistant), estudiantes que no comienzan la agresión, pero participan en ella; y c) agresor reforzador (reinforcer) o estudiantes que animan al agresor y se burlan de la víctima.

2) Víctima, que generalmente se presentan como personas inseguras, sensibles, poco asertivas, físicamente más débiles y con pocas habilidades sociales o amigos. Muchos suelen ser buenos alumnos desde el punto de vista académico (Olweus, 1993). A consecuencia del acoso e incluso del maltrato, pueden sufrir ansiedad, depresión, deseo de no ir al colegio (Kochenderfer y Ladd, 1996) y descenso de rendimiento académico e incluso la aparición de tendencias suicidas. Smith, Talamelli, Cowie, Naylor y Chauhan (2004) clasifican dos subtipos de este rol: a) víctima pasiva (passive victim), típica o sumisa, que se muestra vulnerable, insegura y con baja autoestima; no son acogidas por sus pares y sufren aislamiento social, ya que suelen carecer de habilidades sociales e, incluso, de comunicación. Tal y como observa Díaz-Aguado Jalón (2005), las víctimas pasivas tienden a culpabilizarse de su situación y a negarla, pues la consideran más vergonzosa de lo que consideran su situación los agresores, quienes incluso se muestran orgullosos de su condición. b) Víctima activa (active victim) o "víctima provocadora" en los términos de Díaz-Aguado Jalón (2005), también sufre aislamiento social e impopularidad, discriminación y exclusión. No obstante, su reacción a los abusos se basa en la agresión, conductas irritantes e impulsividad y son incapaces de resolver el conflicto mediante una conducta pasiva; muchas tienen problemas de concentración e incluso hiperactividad. Trautmann $(2008,15)$ denomina a este subtipo de víctimas, bully-víctimas.

3) Testigo, espectador o bystander, son la audiencia del agresor -ya sean pares, profesores, familiares, personal del colegio, etc.- que estimulan o inhiben al agresor; de ahí la importancia de que adopten un rol preventivo (Twenmow, Fonagy y Sacco, 2004). Salmivalli (1999, 454) distingue entre: a) defensor (defender), que ayuda o intenta ayudar a la víctima; b) espectador (outsider), que no se involucra en la situación; y c) personas sin rol definido (no clear role), 
bien porque desconocen la situación bien porque no intervienen a pesar de su conocimiento del maltrato o acoso.

Por otra parte, Celaá $(2011,11)$ establece las siguientes consecuencias del maltrato entre iguales: para el agresor puede ser el estado previo a una conducta delictiva en el futuro; la agresión y las relaciones basadas en el modelo de jerarquía-sumisión pueden convertirse en la forma de obtener poder en la vida adulta e incluso valorar la violencia como un hecho socialmente aceptable y recompensado, y en violencia machista o maltrato. Para la víctima puede suponer el fracaso escolar, un trauma psicológico, un riesgo físico, así como otros problemas de personalidad y riesgo para su desarrollo equilibrado, traducido, por ejemplo, en ansiedad e infelicidad e incluso la interiorización de su rol de víctima. Para los espectadores puede conducir a una actitud pasiva y permisiva ante las injusticias, la desigualdad y a una modelación errónea de la valía personal y de las formas de relación positiva.

Partiendo de estas premisas, analizaremos un caso de aislamiento por parte de un grupo de chicas de tercer ciclo de Primaria a otra chica de acuerdo a los siguientes pasos: en primer lugar, definiremos el contexto donde la situación de un tipo de maltrato escolar (aislamiento) tuvo lugar incluyendo las características del grupo clase así como de las agresoras o marginadoras y la víctima o marginada. En segundo lugar, detallaremos el problema en profundidad, así como analizaremos las medidas que se tomaron tanto con las alumnas (marginadoras y marginada) como con respecto al tutor, al centro y a los padres de dichas estudiantes. Más tarde, ofreceremos un protocolo tanto de prevención como de intervención surgido a partir de la revisión bibliográfica y la propia experiencia. Por último, comentaremos las conclusiones del estudio de caso y propondremos futuras líneas de investigación derivadas del mismo.

\section{Contexto}

La situación de exclusión y marginación social se originó en un colegio público céntrico de una capital de provincia de Castilla-La Mancha que mantendremos en el anonimato con el fin de proteger a todas las partes implicadas. Asimismo cualquier información que pueda identificarlas ha sido omitida o neutralizada en términos más generales. Dicho centro estaba situado en un barrio no conflictivo en el que habitan fundamentalmente familias con un nivel socio-económico medio.

La clase pertenecía al tercer ciclo (quinto y sexto cursos) de Educación Primaria y se componía de veintitrés alumnos, incluidos una niña repitiendo curso y dos niños con síndrome de Asperger. Son alumnos en su mayoría trabajadores y respetuosos, con familias que se preocupan por su rendimiento académico y el desarrollo de las clases. En esta clase hay un chico con el rol de líder, muy inteligente pero, a veces, un poco déspota con sus compañeros y también muestra rechazo a la chica marginada. Los motivos que alega para dicho rechazo es que la niña siempre va detrás de él y él quiere que no lo siga molestando. También hay una estudiante líder de un grupo de chicas numeroso, cuyos resultados académicos son los más altos, que claramente imponen sus opiniones a los demás. En este caso, son las marginadoras, pero la líder sobresale entre ellas y es la que no deja que la marginada forme parte de su grupo, luego convence a 
sus pares para que tampoco estas la acepten. Por último, la chica marginada siempre ha mostrado inhabilidades sociales, pero en los dos últimos cursos de Primaria, la situación ha empeorado. En tercer y cuarto cursos tenía una amiga en el centro, pero al promocionar al siguiente curso, la madre de dicha alumna pidió al colegio que separasen a su hija de clase, por lo que una quedó en el grupo A y la otra en el B. Durante los recreos, su antigua amiga la evita y ella intenta forzar ser parte del grupo nuevo, pero, al no conseguir su objetivo, insulta a las demás y este comportamiento hace que las demás aún la acepten menos. Además, debido a problemas específicos de salud, la marginada toma una medicación muy fuerte que la vuelve irascible y un poco violenta, hecho que su propia madre reconoce. No obstante, en el desarrollo de las clases no se percibe ningún problema ni ningún signo de marginación. Todo está en calma y el ambiente de trabajo es muy bueno y distendido. Además, la marginada no se queja de su aislamiento ni de su exclusión ni marginación social -en términos de Celaá $(2011,10)-$ ni ante sus padres ni ante el profesorado.

\section{Análisis}

A pesar de que no existan quejas por parte de la marginada, el tutor se apercibe de que, durante el recreo, la niña marginada intenta unirse a los grupos de otras alumnas, pero siempre queda aislada. Además, el profesor de educación física comenta que en su clase, cuando hace grupos, nadie la elige; es más, el resto de sus pares se queja de que se incluya en sus grupos.

Un día en concreto, en clase de educación para la ciudadanía y derechos humanos, el tutor habla sobre la marginación en el recreo y ella expresa clara y explícitamente que todos la aborrecen y que todos "le tienen asco" literalmente. Ante este comentario, el tutor pone en marcha un plan de actuación. En primer lugar, el tutor mantiene charlas individuales con todos los alumnos implicados (el chico líder, la chica líder y su grupo de amigas y la marginada). En dicha reunión, la marginadora dice que no quiere unirse a la marginada porque les pega y les insulta. Ante esta contestación, el tutor intenta hacerle comprender que este comportamiento se produce solo porque la marginada quiere formar parte del grupo y, al no conseguirlo, actúa de esta forma violenta y agresiva. Al término de la misma, la marginadora promete mejorar la relación con la chica marginada, pero añade que es su madre la que le recomienda que no establezca ningún tipo de relación con ella. Por otro lado, la marginada dice no querer unirse a la marginadora ni a su grupo pues la insultan aunque reconoce que ella también lo hace e incluso les pega. El tutor le aconseja que cambie de actitud e intente hablar con ellas y no adopte una posición ofensiva ni agresiva.

En segundo lugar, el tutor pasa una encuesta anónima en clase sobre las relaciones entre todos los alumnos sin mencionar a ninguna persona en particular. Algunas de dichas preguntas eran: ¿tienes amigos en clase?, ¿con quién te gusta jugar en el recreo?, ¿hay alguien con quien no te guste jugar?, ¿tus compañeros se portan bien contigo?, ¿alguna vez te han insultado o se han reído de ti? y ¿quiénes son tus mejores amigos en clase? Valoradas las respuestas se obtienen dos datos muy reveladores: por un lado, a pesar de que los dos alumnos con síndrome de Asperger no tienen muchos 
amigos en el grupo, la gran mayoría de estudiantes contestó que no querían jugar con la marginada por encima de los alumnos recién mencionados; y por otro lado, nadie la eligió como buena amiga.

A continuación, el orientador pasa otra encuesta, en este caso, sociométrica ${ }^{1}$ (Díaz Langa y Martínez García, s.f.) sin advertir al alumnado de que hubiese ningún problema. A la pregunta “¿te sientes maltratado por tus compañeros?”, la víctima marginada contestó que sí. Con esos resultados, el tutor vuelve a iniciar la participación activa en el patio de recreo y empieza a hablar con todo el alumnado de esa clase para incluir a la marginada en varios grupos. Estos la aceptan, pero como imposición del tutor. No obstante, se atisba un pequeño avance.

Por otro lado, después de que el tutor y el profesor de la asignatura de educación física hablen, se decide que en esta última asignatura los grupos no se formen de manera libre, sino que sea el profesor el que decida quién conforma cada grupo y así evitar que la víctima marginada se quede aislada. En otras clases impartidas por el tutor, la composición de los grupos para el trabajo en equipo es rotatoria para que se establezca una mejor relación entre toda la clase. No obstante, surge otro problema: la víctima se queja de que otra alumna (segunda marginadora) la aísla, la ignora o la insulta.

Para afrontar la situación, el tutor se reúne con las madres de manera individual y nota que las madres no quieren que ninguna de las tres niñas se relacione entre ellas. El tutor intenta hacerles ver que, aunque no sean amigas, es necesario que mantengan una relación respetuosa. Pero cada madre considera que su hija se comporta bien y culpa a las demás de insultar y marginar; es más, ninguna admite que su hija pueda tener algo que ver en el problema. Después de esto, las tres madres no se dirigen la palabra entre sí y guardan las distancias.

No obstante lo anterior, gracias a la perseverancia del tutor en los recreos, la víctima marginada parece un poco menos aislada y muestra una actitud más alegre. Esto continuó hasta la excursión de final de curso, donde no hubo ningún problema e incluso compartió habitación con niñas que, si bien no mostraban demasiado agrado, tampoco oposición. En un evento realizado a final de curso, la marginada quería participar en una actividad de carácter artístico y creativo que requería habilidades motrices, ritmo y talento en la que también participaría el grupo en el que se insertaban las marginadoras. Esto supuso la nueva intervención del tutor para que la aceptaran, pues a pesar de que el tutor siempre estaba presente, a menudo surgían problemas y dificultades en el transcurso de la preparación de la mencionada actividad y siempre derivaban de la actuación de la niña marginada, que no conseguía seguir el ritmo del

\footnotetext{
${ }^{1}$ Casanova $(1995,139)$ establece que un test sociométrico sirve para detectar las preferencias intelectuales y afectivas del alumnado así como los rechazos que puedan existir dentro del grupo clase. Dicho test sociométrico se basa en tres preguntas clave que recogen información sobre a quién se elige en situaciones de trabajo y estudio, a quién se elige en situaciones de diversión y a quién se rechaza. El alumnado puede ofrecer más de un nombre; así, el educador puede ver el orden de preferencia de las personas elegidas y las que nadie escoge para así prestarles mayor atención.
} 
resto de sus compañeros. Al final, el evento tuvo unos resultados muy satisfactorios para todas las partes (marginada, marginadoras y entorno escolar), pero constituyó un gran esfuerzo por parte de todos los implicados. Así, el curso acabó con una aceptación “obligada” pero suficiente para evitar problemas.

A pesar de todo lo anterior, como no hubo una denuncia por ninguna de las partes, no se inició un protocolo formal institucional. Por tanto, no quedó realmente demostrado que existiese una práctica abusiva o maltrato o acoso escolar. El equipo directivo no se implicó en el tema y el tutor solo encontró el apoyo del orientador del centro, quien pasó las encuestas sociométricas ya mencionadas y las evaluó.

Al curso siguiente, ya en Educación Secundaria, la madre de la niña marginada decidió que su hija cursara sus estudios de ESO en un instituto al que no asistían ni la marginadora líder ni el niño líder que tampoco la soportaba. Pero en dicho instituto, en la misma clase, estaba la segunda chica marginadora, quien -según la víctima marginada- también la insultaba. Ante esta situación, la madre de la marginada acusó a esta segunda alumna de maltrato a su hija y pidió que la cambiasen de grupo, pero no lo hicieron. La situación era tensa pues, incluso, un día las madres de ambas niñas se pelearon delante de la puerta del instituto, donde tuvieron que ser separadas por otras madres allí presentes. A mitad de curso, la madre de la marginada pasó por el antiguo colegio de su hija, para informar al tutor de Educación Primaria sobre la situación y comentó su decisión de cambiarla de instituto para el siguiente curso. La razón que daba para ello era que, aunque la relación con la segunda marginadora, había mejorado un poco, la marginada no era aceptada por el resto de la clase.

Así, a partir de la experiencia relatada, de la propia práctica docente y de la revisión bibliográfica, hemos elaborado un protocolo tanto de prevención como de actuación para posibles casos específicamente de Educación Primaria -e incluso aplicable en Educación Infantil- que pudiesen surgir en el futuro en centros similares.

Tal y como sugiere Trautmann (2008, pp. 15-16) es necesario establecer una taxonomía de intervenciones que prevengan el maltrato entre pares que ofrezcan cierta garantía de resultados. Así, ofrecemos una visión de sus cinco clasificaciones añadiendo nuestra experiencia personal en Educación Primaria. En primer lugar, las intervenciones curriculares, que consisten en fomentar la actitud anti-acoso y la toma de conciencia del bullying desde el aula y ayudar a los niños a desarrollar habilidades para resolver conflictos y adquirir valores sociales, especialmente a través de grupos de discusión o la tutoría (como sucedió en nuestro caso), desde edades tempranas, no únicamente en el último ciclo de Educación Primaria o en Educación Secundaria, como suele ser lo habitual. Igualmente, se promueve la asertividad para disuadir al agresor y la postura activa de los testigos o espectadores. No obstante, suele ser inefectiva, pues es poco probable que una intervención a un solo nivel tenga un verdadero impacto, ya que no implica a toda la comunidad educativa (Smith, Schneider, Smith y Ananiadou, 2004). En segundo lugar, las intervenciones de entrenamiento de habilidades sociales de forma individual, apoyadas en cambios cognitivos y de comportamiento, tienen mejores resultados en los casos de niños más pequeños -como el nuestro, pues además el maltrato es psicológico e incluso no intencionado, pues las marginadoras no querían que se uniese a su grupo, pero su objetivo no era el acoso y persecución de la 
marginada-, pero no en los agresores (Vreeman y Carroll, 2007). En tercer lugar, las intervenciones integrales $\mathrm{y} / \mathrm{o}$ institucionales, que son multidisciplinarias y se realizan a distintos niveles en la organización del colegio, pues asumen el maltrato o el acoso como un problema sistémico que intenta implicar a toda la comunidad educativa; así, el éxito de la intervención radica en el compromiso tanto institucional como de la mencionada comunidad. En cuarto lugar, la tutoría o mentoring, que como King, Vidourek, Davis y McClelland (2002) demostraron, consigue reducir tanto los avisos por maltrato o acoso, las peleas físicas y los sentimientos depresivos, a la vez que proveen a los niños de las habilidades, rudimentos y lenguaje necesarios para la resolución de conflictos de forma pacífica. En quinto lugar, las intervenciones sobre el testigo o espectador, que se incluyen dentro de los programas integrales de concienciación y construcción del entorno escolar como un lugar seguro. El rol del espectador es relevante, pues -como decíamos en el apartado correspondiente al marco teórico- favorece o disminuye la situación de maltrato o acoso. Por ello, Rigby y Johnson (2005) recomiendan motivar a los testigos para que adopten una actitud activa e intenten detener las situaciones de maltrato o acoso, poniendo vídeos en clase que muestren el papel de los testigos en distintos tipos de situaciones ya mencionadas y comentando qué sienten, qué harían y por qué. Incluso se pueden practicar los juegos de rol para que los alumnos experimenten con los sentimientos y puedan poner en práctica la catarsis y la empatía (Salmivalli, 1999, 456). Así, se expone el problema y se ofrecen a la clase pautas de comportamiento para que adquieran las habilidades sociales y los valores apropiados.

Tras los mencionados sistemas de prevención de situaciones de maltrato o de acoso, ofrecemos un protocolo de actuación para abordar dicho tipo de situaciones cuando ya estén ocurriendo basado en las opiniones de Celaá (2011), pero aplicado específicamente a Educación Primaria, y en nuestra propia experiencia que debería tener las siguientes características. En primer lugar, cuando se observa uno de estos conflictos de convivencia, es necesario que se asegure la intervención coordinada y dirigida por el equipo directivo del centro de todos los profesionales de la educación así como del resto de la comunidad educativa. En segundo lugar es imprescindible que se mantenga la confidencialidad de las partes implicadas -tanto de las supuestas víctimas como de los supuestos agresores y, en su caso, testigos- para así protegerlas. Por último, respecto a los procedimientos y protocolos de actuación que los centros han de iniciar para determinar tanto la existencia o inexistencia de una situación de maltrato o acoso así como las intervenciones posteriores han de estar sistematizados de forma que garanticen la eficacia de dichas actuaciones. Por otro lado, se han de realizar intervenciones de urgencia cuando se observe la necesidad de adoptar medidas inmediatas -como la vigilancia, la solicitud de colaboración familiar, las tutorías individualizadas, entre otras- con el objetivo de garantizar la protección de la víctima y sus derechos.

A continuación definimos las fases del protocolo de actuación una vez que un centro percibe un posible caso de maltrato o acoso -ya sea físico, psicológico o verbalentre iguales y tiene constancia de alguna queja o denuncia formal, igualmente basado 
en la guía de Celaá (2011) pero aplicado concretamente a Educación Primaria². En primer lugar, se comunicará la situación al director y al equipo directivo, quienes tomarán la decisión de iniciar una investigación y tomar determinadas medidas en cuantas reuniones estimen oportunas y consultando a los expertos pertinentes así como acotar los plazos y designar a las personas responsables de cada una de las fases. Cuando corresponda, también serán los encargados de avisar y enviar la documentación necesaria a la Inspección Educativa. Si no existe una evidencia probada de maltrato o acoso, se procedería a un tratamiento educativo según el caso. Si, por el contrario, existen pruebas fehacientes y datos objetivos de maltrato o acoso, se iniciaría una investigación que supusiese el recabo de la siguiente información: a) recogida de información complementaria si el asunto lo requiere; b) puesta en marcha de un protocolo de actuación consensuado entre los profesionales de educación, así como en el aula proporcionar pautas de actuación adecuadas y reeducar conductas contrarias a la convivencia, desarrollar las competencias social y ciudadana y la de autonomía e iniciativa personal; c) convocatoria de reuniones con las familias y, en su caso, el resto de la comunidad educativa implicada con el menor número de interlocutores posible y trabajando con las familias primero por separado para después poder trabajar todas en conjunto $^{3}$; d) seguimiento de la evolución de la situación y evaluación del proceso y resultado del conflicto. Este informe, además, se volverá a enviar a la Inspección Educativa. Más adelante, la situación se comunicaría a otras instancias si procediese y se estimase oportuno. Y finalmente, se concluirá el caso. Para todo esto sería necesario que el centro redactase tanto una guía como los documentos necesarios para cada uno de los pasos ya mencionados, que sirva de apoyo a todos los miembros implicados y, además, suponga un compromiso institucional por escrito.

\section{Conclusiones}

Las conclusiones a las que hemos llegado se pueden clasificar en tres tipos: primeramente, las extraídas de la propia experiencia; en segundo lugar, del seguimiento y del comportamiento posterior de las partes implicadas, especialmente de la chica marginada, en Educación Secundaria; y por último, la aplicación de las mismas a trabajos o investigaciones futuros.

\footnotetext{
${ }^{2}$ Esta misma autora $(2011,29)$ recoge los siguientes casos de maltrato: exclusión y marginación social, como ignorar a alguien (pasiva), no dejarle participar (activa) o discriminar por cuestión de raza, sexo, religión, apariencia, discapacidad, etc.; agresión verbal, como insultar (directa), hablar mal de otra persona (indirecta), calificarlos a través de motes (mixta); agresiones físicas indirectas, como esconder cosas a una persona, romperle cosas o robarle cosas; agresiones físicas directas, como golpear a una persona; intimidación, chantaje o amenazas; acoso o abuso sexual o sexista; y, por último, ciberacoso, esto es, amenazar, insultar o reírse de otra persona, entre otros, mediante mensajes, correo electrónico, chat, redes sociales, etc. o grabar situaciones de maltrato, acoso, vejatorias o que violen la intimidad y distribuirlas a otros compañeros o subirlas a internet.

${ }^{3}$ En este tipo de reuniones es importante no culpabilizar a nadie y hablar desde el respeto y la sutileza para sugerir cambios o hacer propuestas, como lo hizo el tutor en la reunión con las madres de nuestro caso.
} 
Así consideramos que es necesario definir con claridad qué se entiende por maltrato en sus diferentes grados y distinguirlo de otras conductas agresivas, violentas, ofensivas o inapropiadas puntuales. Por tanto, hemos de ser conscientes de que no todos los casos de maltrato o acoso escolar revisten la misma gravedad. Igualmente y como se observa en el protocolo de actuación ofrecido en el apartado anterior, la presencia de ciertos rasgos de situaciones de maltrato o acoso no asegura su existencia. No obstante, aunque no compongan un caso de maltrato como tal, estos comportamientos deben ser tratados y corregidos y nunca deben ser escondidos, como a veces ocurre por temor o miedo.

Como se aprecia en el caso comentado, el maltrato no es siempre una agresión física, una actitud ofensiva o el insulto explícito, sino que ignorar a alguien o no dejarle participar ya sea dentro del aula como en los espacios y tiempos de recreo también puede ser un tipo de maltrato o acoso escolar; además, este comportamiento es mucho más usual entre pares femeninos.

Asimismo estimamos que las soluciones a este tipo de problemas requieren los esfuerzos de toda la comunidad educativa y que la actuación única del tutor puede no ser suficiente. No obstante, la labor tutorial y el tratamiento tanto grupal como individual del alumnado es fundamental para prevenir o solucionar cualquier problema de esta índole. El rol de las familias en estas situaciones es clave, ya que a veces son las que propician la conversión de un alumno en agresor o son los testigos o espectadores que estimulan al agresor. Por ello conviene trabajar con ellas y por separado, para así evitar enfrentamientos, y dejar muy claro desde el principio de las reuniones que el último objetivo es el bienestar y el desarrollo adecuado de sus propios hijos, esto es, del alumnado del centro. Finalmente a este respecto consideramos que es necesario iniciar un protocolo de intervención cuando existe una denuncia, pruebas fehacientes o datos objetivos de algún tipo de situación de maltrato o acoso escolar; pero también es primordial que cuando se detecte un caso de esta clase se realice una intervención pedagógica de oficio aunque no exista ninguna denuncia expresa.

En cuanto a las actitudes y comportamientos de las partes implicadas se pueden extraer las siguientes conclusiones. Creemos que las marginadoras, especialmente la líder, no eran agresoras en los términos que describimos en el marco teórico, pues si bien asumieron que su conducta no era la más adecuada, en ningún momento creyeron que podían estar maltratando a la niña marginada. Esto es, no eran conscientes de que el hecho de aislar o marginar a esta niña fuese una conducta reprochable y negativa. Simplemente, la líder tenía sus amigas y no quería que ella formara parte del grupo. Así, con las charlas mantenidas con el tutor, la niña reflexionó y decidió que, aunque no fueran amigas fuera del entorno escolar, dentro eran todas compañeras, además, de la misma clase. Por otra parte, la marginada cambió su conducta y, en vez de actuar como víctima activa -en términos de Díaz-Aguado Jalón (2005)- al insultar y pegar cuando alguien no quería jugar con ella, intentaba al menos dialogar e integrarse para que la aceptaran en el grupo. Ninguno de los padres de las partes implicadas volvió a presentar ninguna otra queja.

No obstante, en el episodio relatado en el análisis y sucedido cuando la segunda marginadora y la niña marginada cursaban Educación Secundaria muestra que, a veces, 
los propios familiares -en este caso, las madres- fomentan el enfrentamiento de los hijos e incluso lo explicitan llegando a los insultos directos o la violencia física, como efectivamente sucedió. Esto demuestra que la actitud de los padres se refleja en el comportamiento de los hijos. Por otro lado, se constata que cuando la víctima es activa realmente adopta dos roles a la vez: el de víctima, pero también el de agresor, pues reacciona de forma violenta ya sea verbal o física. Por ello, llegamos a la conclusión de que en estos casos, aunque los marginados cambien de centro, no solucionan este problema. Es más, en el caso comentado, el cambio al centro de Secundaria agravó la situación. Por ello, es conveniente un cambio de actitud y trabajar sus habilidades sociales, especialmente durante las tutorías, ya que les harían mejorar sus relaciones en el futuro y los ayudarían a una mejor integración en grupos. Igualmente, un seguimiento de estos alumnos y la colaboración entre los maestros y profesores de distintas etapas educativas e incluso de distintos centros a los que estos alumnos asistan serían necesarios para abordar y erradicar este tipo de situaciones en su totalidad. Una de las limitaciones de nuestro estudio es que solo pudimos tratar el caso en la etapa de Educación Primaria, donde impartíamos docencia, pero no en Educación Secundaria, pues los alumnos ya no pertenecían a nuestro centro.

En cuanto al rol del tutor en el caso, este refiere en su propia autoevaluación que hubo aspectos positivos, como el tratamiento grupal e individual tanto de la víctima como del resto de alumnas marginadoras, ya que se consiguió una aceptación obligada pero efectiva que permitió la convivencia pacífica y redujo el sufrimiento de la víctima. Igualmente considera positiva su intervención en el patio de recreo intentando integrar a la víctima en distintos grupos y observando sus reacciones. El trabajo grupal dentro del aula también constituyó una experiencia positiva, pues la alumna tuvo la posibilidad de relacionarse con el grupo de marginadoras en un entorno protegido, donde se sentía segura. Sin embargo, el tutor refiere algunos aspectos que podrían haberse mejorado. Por ejemplo, una mayor implicación del equipo directivo y del equipo docente, pues las familias habrían tenido una actitud más correcta si hubiesen tenido que enfrentarse a todo equipo del centro y no únicamente al tutor. Asimismo, la falta de información sobre este tema, le hizo abordar la situación de una manera personal, lo que podría haber mejorado mucho si hubiese tenido acceso a un protocolo de actuación.

Por los motivos arriba mencionados creemos que sería conveniente seguir investigando en los roles de los partícipes de las situaciones de maltrato o acoso escolar. Pero no incidir tanto en los tipos de agresor o testigos -comentados en el marco teórico de este estudio- pues ya existen numerosos estudios que los abordan igualmente tratados en la introducción y en el desarrollo de este trabajo-, sino en cómo abordar a la víctima así como compendiar un conjunto de sugerencias o consejos que puedan ayudarle tanto a solucionar el problema como a prevenirlo. Ello no solo implicaría la labor docente e investigadora, sino pedagógica, psicológica, parental e institucional. 


\section{Referencias bibliográficas}

ALBALADEJO-BLÁZQUEZ, N, FERRER-CASCALES, R., REIG-FERRER, A. y FERNÁNDEZ-PASCUAL, M. D. (2013). ¿Existe violencia escolar en Educación Infantil y Primaria? Una propuesta para su evaluación y gestión. Anales de Psicología, 29(3), 1069-1069.

ALSAKER, F. D. y NAGELE, C. (2008). Bullying in Kindergarten and Prevention, en W. Craig y D. Pepler (eds.), International Perspective on Understanding and Addressing Bullying, 230-252. Kingston: PREVNet.

ALSAKER, F. D. y VILÉN, U. L. (2010). El bullying en la edad preescolar, en R. Ortega (ed.), Agresividad injustificada, bullying y violencia escolar, 129-164. Madrid: Alianza Editorial.

AVILÉS MARTÍNEZ, J. M. (2006). Bullying: El maltrato entre iguales. Agresores, víctimas y testigos en la escuela. Salamanca: Amarú Ediciones.

AVILÉS MARTÍNEZ, J. M. y MONJAS CASARES, I. (2005). Estudio de incidencia de la intimidación y el maltrato entre iguales en la educación secundaria obligatoria mediante el cuestionario CIMEI (Avilés, 1999) -Cuestionario sobre Intimidación y Maltrato Entre Iguales-. Anales de psicología, 1(21), 27-41.

BRIGHI, A., GUARINI, A., MELOTTI, G., GALLI, S. y GENTA, M. L. (2012). Predictors of Victimisation Across Direct Bullying, Indirect Bullying and Cyberbullying. Emotional Behavioural Difficulties, 17, 375-388.

CARRETERO BERMEJO, R. (2011). Sexo, sexismo y acoso escolar entre iguales. Revista Complutense de Educación, 22(1), 27-43.

CASANOVA, M. A. (1995). Un modelo evaluador y su metodología. El camino para llegar a la meta, en M. A. Casanova, Manual de evaluación educativa, 120-180. Madrid: La Muralla.

CELAÁ, I. (2011). Guía de actuación en los centros educativos ante el maltrato entre iguales.

http://www.hezkuntza.ejgv.euskadi.net/r43detail/es/contenidos/informacion/dif11/es_5613/adjuntos/malos_tratos/tratu_txarrak _2011_c.pdf 3/12/2013.

CHAGAS DORREY, R. (2005). Los maestros frente a la violencia entre alumnos. Revista Mexicana de Investigación Educativa, 10, 1071-1082.

DÍAZ-AGUADO JALÓN, M. J. (2005). Aprendizaje cooperativo y curriculum de la no-violencia, en M. J. Díaz-Aguado Jalón, Mejorar la convivencia en el aula a través del aprendizaje cooperativo y el currículum de la no-violencia. http://mariajosediaz-aguado.blogspot.com.es/2005/07/aprendizaje-cooperativo-ycurriculum.html 19/02/2014.

DÍAZ-AGUADO JALÓN, M. J., MARTÍNEZ ARIAS, R. y MARTÍN SEOANE, G. (2004). Prevención de la violencia y lucha contra la exclusión desde la adolescencia. La violencia entre iguales en la escuela y en el ocio (Volumen 1). Madrid: Instituto de la Juventud. 
DÍAZ LANGA, E. y MARTÍNEZ LANGA, M. (s.f.). Batería Psicopedagógica EOS 6. Ámbito óptimo de utilización: $6^{\circ}$ Curso de EDUCACIÓN PRIMARIA. Versión 1.0: Instituto de Orientación Psicológica EOS.

FREEDMAN, J. (2000). Easing the Teasing: How Parents can Help their Kids Cope. Winnetka Alliance for Early Childhood, 1-4.

FENAUGHTY, J y HARRÉ, N. (2013). Factors Associated with Distressing Electronic Harassment and Cyberbullying. Computers in Human Behavior, 29, 803-811.

GARAIGORDOBIL, M. y ALIRI, J. (2013). Ciberacoso (“cyberbullying”) en el País Vasco: Diferencias de sexo en víctimas, agresores y observadores. Behavioral Psychology/Psicología Conductual, 21(3), 461-474.

HARO-SOLÍS, I, GARCÍA-CABRERO, B y REIDL-MARTÍNEZ, L. M. (2013). Experiencias de culpa y vergüenza en situaciones de maltrato entre iguales en alumnos de Secundaria. Revista Mexicana de Investigación Educativa, 18(59): 1047-1075.

KING, K. A., VIDOUREK, R. A., DAVIS, B. y MCCLELLAN, W. (2002). Increasing Self-esteem and School Connectedness through a Multidimensional Mentoring Program. Journal of School Health, 72(7), 294-299.

KOCHENDERFER, B. J. y LADD, G. W. (1996). Peer Victimization: Cause or Consequence of School Maladjustment? Child Development, 67(4), 1306-1317.

MONKS, C. P., PALERMITI, A., ORTEGA, R. y COSTABILE, A. (2011). A CrossNational Comparison of Aggressors, Victims and Defenders in Pre-Schools in England, Spain and Italy. The Spanish Journal of Psychology, 14(1), 133-144.

OLENIK-SHEMESH, D., HEIMAN, T. y EDEN, S. (2012). Cyberbullying Victimisation in Adolescence: Relationships with Loneliness and Depressive Mood. Emotional and Behavioural Difficulties, 17, 361-374.

OLWEUS, D. (1993). Bullying at School: What we Know and What we can Do. Oxford: Blackwell Publishing.

OLWEUS, D. (1998). Conductas de acoso y amenaza entre escolares. Madrid: Ediciones Morata.

RAMÍREZ-LÓPEZ, C. A. y ARCILA-RODRÍGUEZ, W. O. (2013). Violencia, conflicto y agresividad en el escenario escolar. Educación y Educadores, 16(3): 411-429.

RIGBY, K. y JOHNSON, B. (2005). Student Bystanders in Australian Schools. The Journal for Pastoral Care \& Personal-Social Education, 23(2): 10-16.

ROSS, D. M. (1996). Childhood Bullying and Teasing. What School Personnel, other Professionals, and Parents can Do. Alexandria, VA: American Counseling Association.

SALMIVALLI, C. (1999). Participant Role Approach to School Bullying: Implications for Interventions. Journal of Adolescence, 22, 453-459. 
SMITH, P. K., TALAMELLI, L., COWIE, H., NAYLOR, P. y CHAUHAN, P. (2004). Profiles of Non-Victims, Continuing Victims and New Victims of School Bullying. British Journal of Educational Psychology, 74, 565-581.

SMITH, J. D., SCHNEIDER, B. H., SMITH, P. K. y ANANIADOU, K. (2004). The Effectiveness of Whole-School Antibullying Programs: A Synthesis of Evaluation Research. School Psychology Review, 4(33), 547-560.

THERON, W., MATTHEE, D., STEEL, H. y MARTÍN RAMÍREZ, J. (2001). Direct and Indirect Aggression in Women: A Comparison between South African and Spanish University Students, en J. Martín Ramírez y D. Richardson (eds.), CrossCultural Approaches to Aggression and Reconciliation, 99-109. Huntington, NY: Nova Science Publishers.

TOLDOS, M. P. (2005). Sex and Age Differences in Self-Estimated Physical, Verbal and Indirect Aggression in Spanish Adolescents. Aggressive Behavior, 31, 13-23.

TRAUTMANN, A. (2008). Maltrato entre pares o bullying. Una visión actual. Revista chilena de pediatría, 1(79), 13-20.

TWEMLOW, S. W., FONAGY, P. y SACCO, F. C. (2004). The Role of the Bystander in the Social Architecture of Bullying and Violence in School and Communities. Annals New York Academy of Sciences, 215-232.

VREEMAN, R. C. y CARROLL, A. E. (2007). A Systematic Review of School-Based Interventions to Prevent Bullying. Archives of Pediatrics \& Adolescent Medicine, 161(1), 78-88.

WADE, A. y BERAN, T. (2011). Cyberbullying: The New Era of Bullying. Canadian Journal of School Psychology, 26, 44-61.

\section{Correspondencia con los autores}

Raquel SÁNCHEZ RUIZ

Facultad de Educación de Albacete

Plaza de la Universidad 3, 02071, Albacete

e-mail: Raquel.SanchezRuiz@uclm.es

Teléfono: 967-599200; Ext: 2292

Teresa ESCRIBANO LARA

Facultad de Educación de Albacete

Plaza de la Universidad 3, 02071, Albacete

e-mail: Teresa.Escribano@uclm.es

Teléfono: 967-599200; Ext: 96206 